\title{
Tratamento cirúrgico da coarctação do arco aórtico em adulto: avaliação clínica e angiográfica tardia da técnica extra-anatômica
}

\author{
Luiz Augusto F. LISBOA *, Carlos Alberto C. ABREU FILHO *, Luís Alberto O. DALLAN *, \\ Carlos E. ROCHITTE *, Januário M. de SOUZA **, Sérgio Almeida de OLIVEIRA ${ }^{\star \star * \star}$
}

\begin{abstract}
Lisboa L A F, Abreu Filho C A C, Dallan L A O, Rochitte C E, Souza J M, Oliveira S A - Tratamento cirúrgico da coarctação do arco aórtico em adulto: avaliação clínica e angiográfica tardia da técnica extra-anatômica. Rev Bras Cir Cardiovasc 2001; 16(3): 187-94.
\end{abstract}

RESUMO: Objetivo: Avaliar os resultados tardios da técnica extra-anatômica para correção da coarctação do arco aórtico em adultos.

Casuística e Métodos: Entre 1979 e 2000, 15 pacientes foram submetidos à interposição de tubo de Dacron entre a aorta ascendente e descendente para correção da coarctação do arco aórtico. Onze $(73,3 \%)$ pacientes eram do sexo masculino e a média de idade foi de $30,8 \pm 12,1$ anos (18 a 61 anos). A esternotomia mediana, com abordagem do pericárdio posterior, foi utilizada em $13(86,7 \%)$ pacientes e a toracotomia lateral esquerda em $2(13,3 \%)$. Em $3(20,0 \%)$ pacientes foram realizados procedimentos associados e $4(26,7 \%)$ eram reoperações. Os pacientes foram acompanhados por um período de 30 dias a 21 anos (média de 6,9 \pm 6,7 anos) com avaliação clínica, ecocardiográfica e estudo angiográfico com ressonância magnética.

Resultados: Não ocorreram complicações neurológicas e nem mortalidade imediata ou tardia. Não houve necessidade de reoperações e nem complicações tardias com o enxerto. Todos os pacientes encontram-se assintomáticos e com o enxerto pérvio confirmado pelo ecocardiograma. Cinco $(33,3 \%)$ pacientes apresentam hipertensão arterial sistêmica de grau leve. Em 11 (73,3\%) pacientes foi realizado o estudo angiográfico com ressonância magnética, mostrando enxerto com bom funcionamento a longo prazo.

Conclusão: A técnica extra-anatômica, com interposição de tubo de Dacron entre a aorta ascendente e descendente, para correção da coarctação do arco aórtico é uma operação segura, com baixa morbidade e mortalidade. Os bons resultados a longo prazo mostram que esta técnica pode ser uma alternativa segura e menos invasiva, para a correção de coarctação do arco aórtico ou recoarctação em adultos.

DESCRITORES: Coartação aórtica, cirurgia. Aorta torácica, anormalidades. Coartação aórtica, radiografia. Aorta torácica, cirurgia. Aorta torácica, radiografia. Angiografia coronária.

\footnotetext{
Trabalho realizado no Instituto do Coração do Hospital das Clínicas da Faculdade de Medicina da Universidade de São Paulo e no Hospital Beneficência Portuguesa de São Paulo. São Paulo, SP, Brasil.

Apresentado ao 28을 Congresso Nacional de Cirurgia Cardíaca. Ouro Preto, MG, 5 a 7 de abril de 2001.

Laureado com o "Prêmio Nacional de Cirurgia Cardíaca - 2000"

* Do Instituto do Coração do Hospital das Clínicas da Faculdade de Medicina da Universidade de São Paulo.

** Do Hospital Beneficência Portuguesa de São Paulo.

Endereço para correspondência: Luiz Augusto Ferreira Lisboa. Divisão Cirúrgica do Instituto do Coração (InCor) HC-FMUSP. Av. Dr. Enéas de Carvalho Aguiar, 44 - São Paulo, SP, Brasil. CEP : 05403-001. Tel.: (11) 3069-5014. Fax: (11) 3069-5415. e-mail: dcilisboa @ incor.usp.br.
} 
Lisboa L A F, Abreu Filho C A C, Dallan L A O, Rochitte C E, Souza J M, Oliveira S A - Tratamento cirúrgico da coarctação do arco aórtico em adulto: avaliação clínica e angiográfica tardia da técnica extra-anatômica. Rev Bras Cir Cardiovasc 2001; 16(3): 187-94.

\section{INTRODUÇÃO}

A coarctação da aorta localiza-se, com maior freqüência, distal à artéria subclávia esquerda (1). Em geral, é diagnosticada e tratada na infância. A primeira correção cirúrgica, com sucesso, de coarctação da aorta foi realizada em $1945^{(2)}$. Nesse período, várias técnicas foram desenvolvidas e, atualmente, o tratamento cirúrgico da coarctação da aorta está bem estabelecido, apresentando baixa morbidade e mortalidade.

Em pacientes adultos com formas anatômicas atípicas de coarctação da aorta, coarctação do arco aórtico, recoarctação ou com doenças cardíacas associadas, a correção cirúrgica anatômica apresenta maior dificuldade técnica com conseqüente aumento da morbidade e mortalidade. Várias técnicas extra-anatômicas têm sido descritas para simplificar a operação, tais como interposição de enxertos com anastomose proximal na aorta ascendente ou na artéria subclávia esquerda e anastomose distal na aorta abdominal infra-renal ou supra-celíaca $(3,4)$.

Em 1981, OLIVEIRA et al. (5) propuseram uma variante técnica da operação extra-anatômica com interposição de um tubo de Dacron entre a aorta ascendente e a aorta torácica descendente via esternotomia para correção cirúrgica da coarctação do arco aórtico, evitando o manuseio da região de coarctação e simplificando a técnica.

Neste trabalho são avaliados os resultados clínicos e angiográficos, a longo prazo, da técnica extra-anatômica para correção cirúrgica da coarctação do arco aórtico em adultos.

\section{CASUÍSTICA E MÉTODOS}

Entre 1979 e 2000, 15 pacientes com idade entre 18 e 61 anos (média de 30,8 $\pm 12,1$ anos) foram submetidos à operação extra-anatômica para correção de coarctação do arco aórtico. Em 2 pacientes a coarctação localizava-se na região proximal do arco aórtico (entre o tronco braquiocefálico e a artéria carótida esquerda), em 6 localizava-se na região distal (entre a artéria carótida esquerda e artéria subclávia esquerda) e em 7 localizava-se na região do ístmo (entre a artéria subclávia esquerda e a inserção do ducto arterioso).

Todos os pacientes apresentavam hipertensão arterial sistêmica e faziam uso de pelo menos uma medicação anti-hipertensiva. Três $(20,0 \%)$ pacientes apresentavam doenças cardíacas associadas, sendo $1 \mathrm{com}$ dissecção da aorta tipo $A, 1$ com insuficiência da valva aórtica e outro com bloqueio atrioventricular total. Estes pacientes foram submetidos à correção da coarctação da aorta e da doença cardíaca associada no mesmo tempo cirúrgico. Outros $4(26,7 \%)$ pacientes já haviam sido submetidos à correção cirúrgica da coarctação da aorta por toracotomia esquerda e apresentavam recoarctação. Todos os pacientes realizaram ecoDopplercardiografia e 13 pacientes, também, realizaram cateterismo cardíaco com aortografia pré-operatório. O gradiente da coarctação da aorta variou de 45 a $80 \mathrm{mmHg}$ (média de $62,5 \mathrm{mmHg}$ ) na cineangiografia préoperatória e de 40 a $75 \mathrm{mmHg}$ (média de 57,5 $\mathrm{mmHg}$ ) com o ecoDopplercardiograma.

\section{Indicações para a Realização da Técnica Extra-Anatômica}

A técnica extra-anatômica com interposição do tubo de Dacron entre a aorta ascendente e descendente está indicada na coarctação atípica da aorta, coarctação do arco aórtico, ou nas recoarctações. A técnica extra-anatômica com abordagem por esternotomia tem a vantagem da não manipulação da região coarctada, onde há intensa circulação colateral, e de evitar a retoracotomia esquerda nos casos de reoperação além de permitir, quando houver, a correção simultânea de outras doenças cardíacas associadas.

\section{Técnica Operatória}

A via de acesso por esternotomia e circulação extracorpórea estabelecida por canulação da aorta ascendente e drenagem com cânula única de átrio direito e veia cava inferior foi realizada em 13 (86,7\%) pacientes. As anastomoses do tubo de Dacron na aorta ascendente e na aorta torácica descendente foram realizadas com pinçamento lateral da aorta, sem parada cardíaca e com normotermia. O tempo médio de circulação extracorpórea foi de 82 minutos (40 a 190 minutos), sendo de 55 minutos (40 a 70 minutos) entre os pacientes submetidos à correção isolada da coarctação do arco aórtico.

A aorta torácica descendente foi exposta por abordagem do pericárdio posterior, tracionando-se o coração cefalicamente e incisando longitudinalmente o pericárdio posterior (Figura 1). Durante esse procedimento devemos ter cuidado especial com o esôfago, pela sua proximidade com a aorta torácica descendente.

Após o pinçamento lateral da aorta torácica descendente, foi realizada a anastomose distal término - lateral com o enxerto de Dacron, sendo utilizada sutura contínua com fio de polipropileno 40 (Figura 2). Após o término da anastomose distal, o enxerto foi posicionado à esquerda, lateralmente ao ventrículo esquerdo e sobre a artéria pulmonar (Figura 3). Em seguida, o enxerto foi cortado obli- 
Lisboa L A F, Abreu Filho C A C, Dallan L A O, Rochitte C E, Souza J M, Oliveira S A - Tratamento cirúrgico da coarctação do arco aórtico em adulto: avaliação clínica e angiográfica tardia da técnica extra-anatômica. Rev Bras Cir Cardiovasc 2001; 16(3): 187-94.

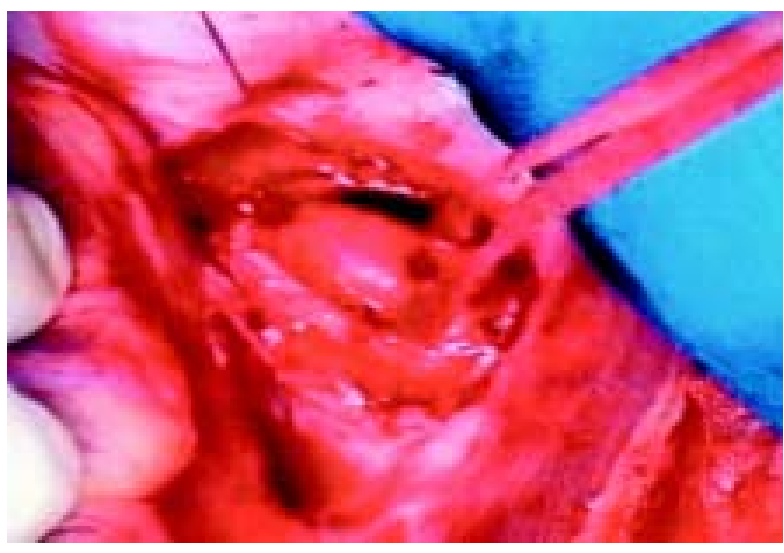

Fig. 1 - Visão intra-operatória mostrando e aorta torácica descendente exposta por abordagem do pericárdio posterior, estando o coração tracionado cefalicamente.

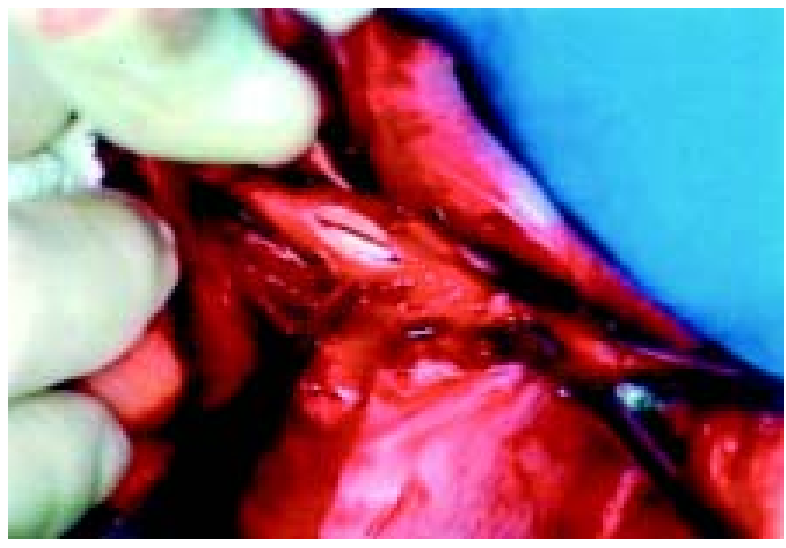

Fig. 2 - Visão intra-operatória mostrando a aorta torácica descendente pinçada laterlamente e incisada longitudinalmente para a realização da anastomose com o tubo de Dacron.

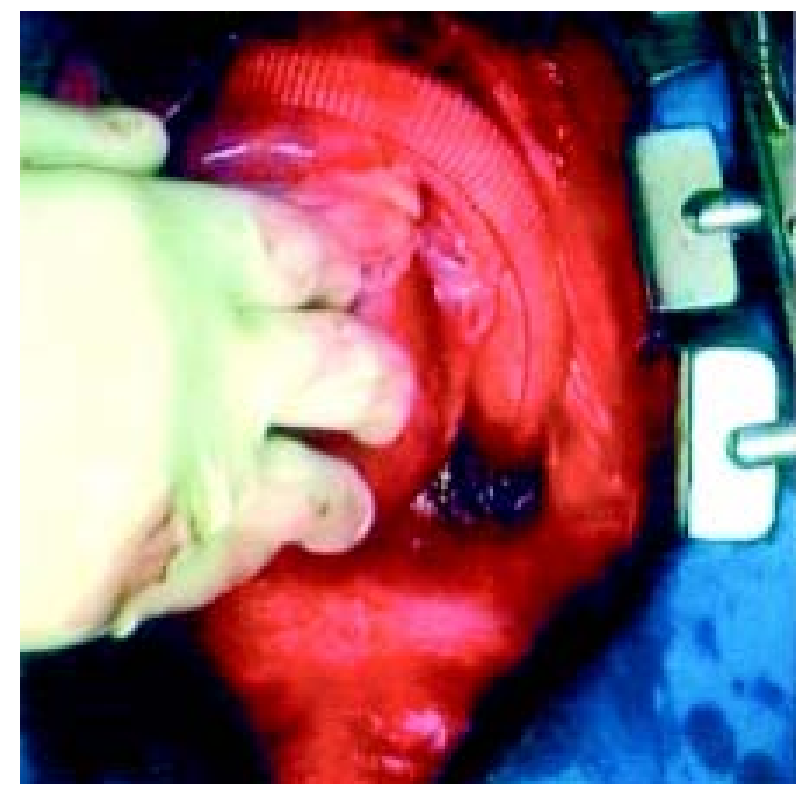

Fig. 3 - Visão intra-operatória mostrando o tubo de Dacron anastomosado na aorta torácica descendente e posicionado à esquerda, lateralmente ao ventrículo esquerdo.

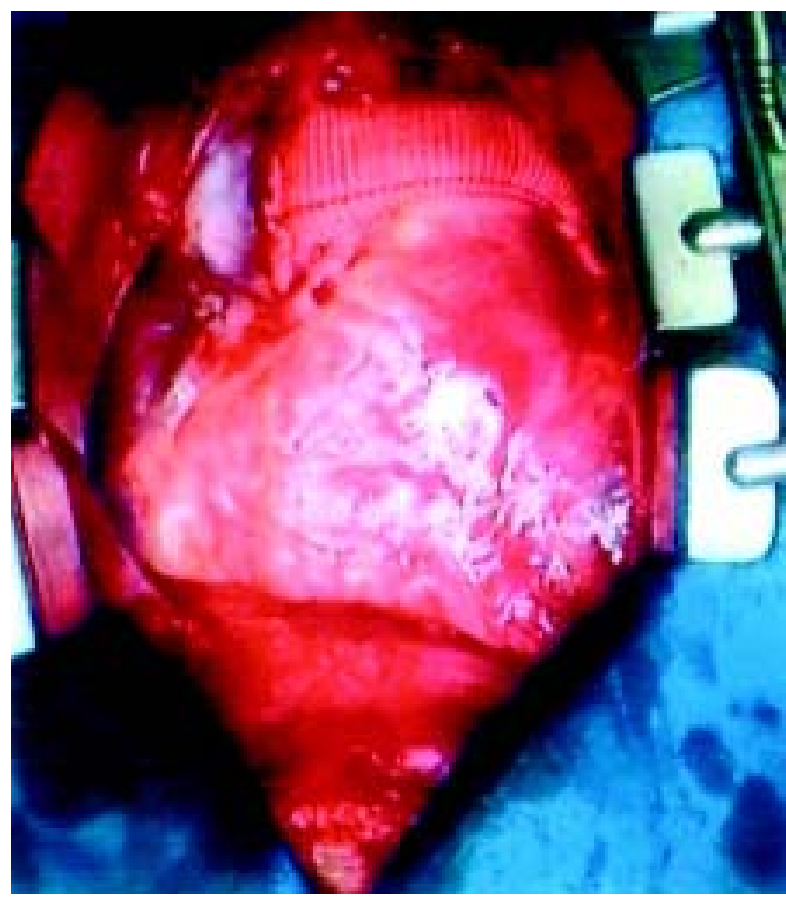

Fig. 4 - Visão intra-operatória mostrando o enxerto extra-anatômico, com interposição do tubo de Dacron entre a aorta ascendente e aorta torácica descendente, passando sobre a artéria pulmonar e lateralmente ao ventrículo esquerdo.

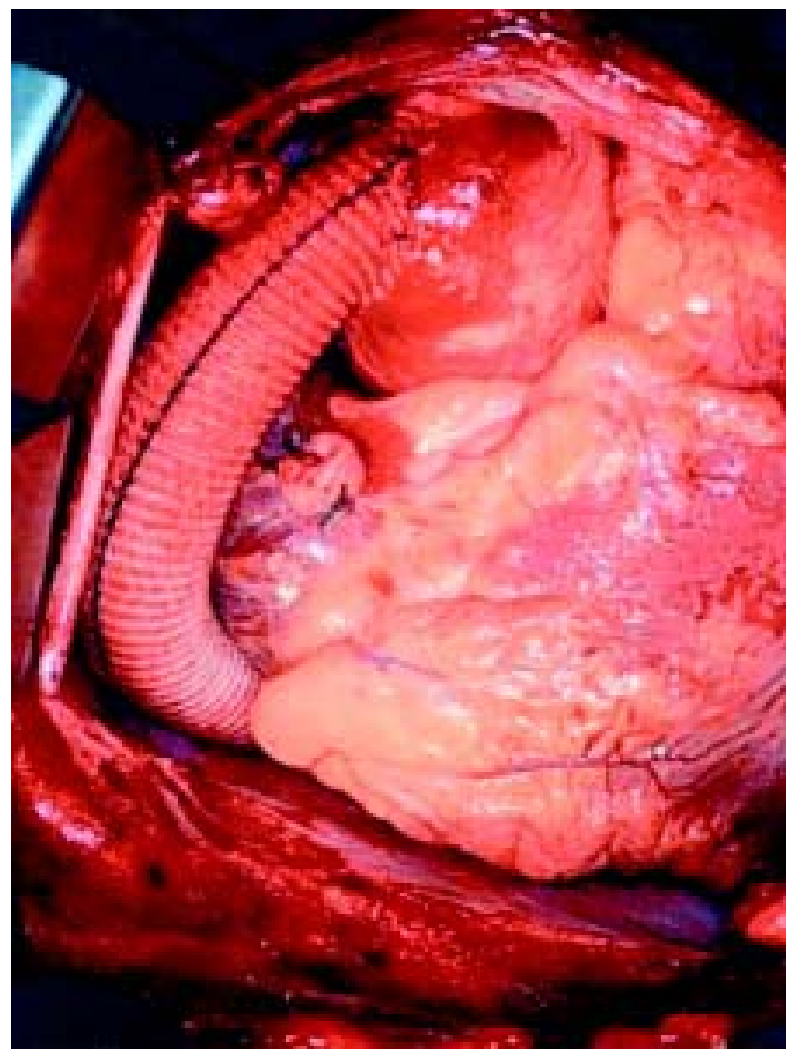

Fig. 5 - Visão intra-operatória mostrando o enxerto extra-anatômico, com tubo de Dacron da aorta ascendente para aorta torácica descendente, passando lateralmente ao átrio direito e por sobre a veia cava inferior. 
Lisboa L A F, Abreu Filho C A C, Dallan L A O, Rochitte C E, Souza J M, Oliveira S A - Tratamento cirúrgico da coarctação do arco aórtico em adulto: avaliação clínica e angiográfica tardia da técnica extra-anatômica. Rev Bras Cir Cardiovasc 2001; 16(3): 187-94.

quamente e realizada anastomose proximal término - lateral na aorta ascendente, utilizando-se pinçamento lateral da aorta e com sutura contínua de fio de polipropileno 4-0 (Figura 4). Do mesmo modo, após o término da anastomose distal, o enxerto pode ser posicionado à direita sobre a veia cava inferior e lateral ao átrio direito sendo anastomosado, com a mesma técnica, na aorta ascendente (Figura 5).

Em $2(13,3 \%)$ pacientes com coarctação na região do ístmo, a via de acesso utilizada foi a toracotomia látero-posterior esquerda. No intra-operatório observou-se o envolvimento da artéria subclávia esquerda na zona de coarctação, sendo realizada a correção cirúrgica pela técnica extraanatômica. O tubo de Dacron foi anastomosado, proximalmente, na aorta ascendente e, distalmente, na aorta torácica descendente, com pinçamento lateral da aorta. A circulação extracorpórea estabelecida através da artéria e veia femoral foi utilizada em um desses pacientes, sendo o outro operado sem circulação extracorpórea.

Os enxertos utilizados para a correção extraanatômica foram tubos de Dacron variando entre 16 $\mathrm{mm}$ e $25 \mathrm{~mm}$ diâmetro (média de $20 \mathrm{~mm}$ ), dependendo do diâmetro da aorta torácica descendente.

\section{Acompanhamento Clínico Pós-Operatório}

Após a alta hospitalar os pacientes foram encaminhados a seus respectivos cardiologistas. Todos os pacientes tiveram avaliações clínicas e ecocardiográficas freqüentes ao longo dos anos. Entre outubro e dezembro de 2000 , todos os pacientes foram contatados, via telefone, pela equipe cirúrgica e seus prontuários revisados. Foram analisadas complicações tardias, o quadro clínico atual, medicação em uso, os ecocardiogramas, necessidade de reoperação, qualidade de vida e mortalidade. Onze $(73,3 \%)$ pacientes deram consentimento e foram submetidos ao estudo angiográfico com ressonância magnética. Quatro pacientes assintomáticos e com excelente qualidade de vida não realizaram o estudo angiográfico, 2 por problemas pessoais e 2 por residirem fora do estado de São Paulo. O tempo médio de seguimento desses pacientes foi de $6,9 \pm 6,7$ anos, variando entre 30 dias e 21 anos.

\section{Métodos de Ressonância Magnética}

Os estudos de ressonância magnética foram realizados em um aparelho GE de $1.5 \mathrm{~T}$, com gradientes de alta performance, especialmente desenvolvido para a geração de imagens cardiovasculares de alta resolução $(\mathrm{CV} / \mathrm{i})$.
As seqüências de pulso utilizadas foram:

O gradiente eco tridimensional - desenhada especialmente para a realização de angioressonância com injeção de bolus intravenoso de contraste paramagnético (gadolínio-DTPA).

A técnica de contraste de fase (cine-ressonância e gradiente eco segmentado) - Essa técnica foi usada para medir a velocidade de fluxo sangüíneo, calcular o volume de sangue na aorta e estimar o gradiente pressórico na aorta torácica descendente após a anastomose do tubo.

Após a aquisição das imagens fontes da angioressonância, estas foram processadas para obtenção de reconstrução tridimensional com técnica de "maximum intensity projection" (MIP) e "surface" (superfície). As imagens pela técnica de contraste de fase foram analisadas com software desenhado especificamente para análise de fluxo [fluxo $\left(\mathrm{cm}^{3}\right)=$ área de secção transversal do vaso $\left(\mathrm{cm}^{2}\right) \times$ velocidade de fluxo $\left.(\mathrm{cm} / \mathrm{s})\right]$ e de gradiente, que usa a fórmula de Bernoulli simplificada $\left(? \mathrm{P}=4 \times \mathrm{V}^{2}\right)$.

\section{RESULTADOS}

\section{Pós-operatório Imediato}

Não houve mortalidade hospitalar e nem complicações neurológicas. Nenhum paciente foi reoperado por sangramento. As complicações pósoperatórias estiveram relacionadas à infecção pulmonar em 2 pacientes e arritmia ventricular em 1 paciente. Esses pacientes apresentaram boa evolução clínica. O tempo de internação variou de 6 a 28 dias (média de 10,2 $\pm 5,8$ dias), e entre os pacientes com procedimento isolado para correção da coarctação do arco aórtico variou de 7 a 15 dias (média de 8,6 \pm 2,6 dias).

\section{Seguimento Clínico Tardio}

Com seguimento clínico pós-operatório de 30 dias a 21 anos (média de 6,9 \pm 6,7 anos), todos os pacientes encontram-se assintomáticos, realizam atividades físicas habituais e apresentam excelente qualidade de vida. Dez $(66,7 \%)$ pacientes estão normotensos (pressão arterial sistêmica sistólica $\leq$ $140 \mathrm{mmHg}$ e diastólica $\leq 90 \mathrm{mmHg})$. Cinco $(33,3 \%)$ pacientes apresentam hipertensão arterial sistêmica de grau leve e em uso de apenas medicação antihipertensiva (3 pacientes em uso de betabloqueador, 1 paciente em uso de inibidor da enzima conversora da angiotensina e 1 paciente em uso de diurético). 
Lisboa L A F, Abreu Filho C A C, Dallan L A O, Rochitte C E, Souza J M, Oliveira S A - Tratamento cirúrgico da coarctação do arco aórtico em adulto: avaliação clínica e angiográfica tardia da técnica extra-anatômica. Rev Bras Cir Cardiovasc 2001; 16(3): 187-94.

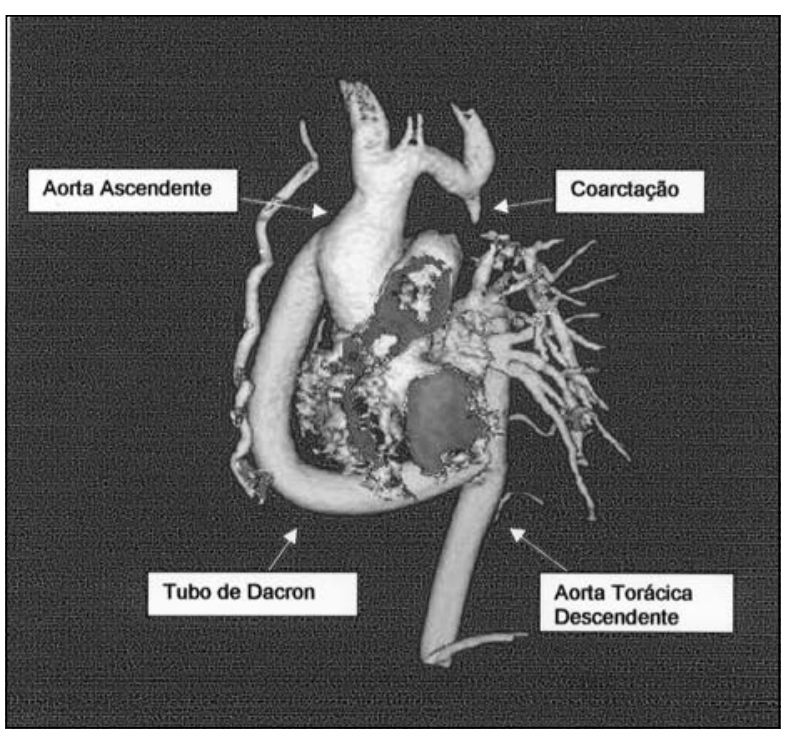

Fig. 6 - Angioressonância (pós-operatório 5 dias) de correção de coarctação do istmo e hipoplasia do arco aórtico, pela técnica extra-anatômica com tubo de Dacron da aorta ascendente para aorta torácica descendente, passando lateralmente ao átrio direito e por sobre a veia cava inferior.

Não ocorreram complicações tardias com o enxerto de Dacron e nem necessidade de reoperação. O estudo com ecocardiograma mostrou enxerto pérvio em todos os pacientes.

\section{Estudo com Ressonância Magnética}

Onze $(73,3 \%)$ pacientes realizaram estudo angiográfico com ressonância magnética entre 5 dias e 21 anos de pós-operatório (média de 7,3 \pm 7,1 anos). A angioressonância da aorta mostrou os enxertos de Dacron pérvios e com estrutura preservada a longo prazo (Figuras 6 e 7). O cálculo da velocidade de fluxo sangüíneo e o volume de sangue na aorta torácica descendente, após a anastomose com o tubo de Dacron, mostrou valores fisiológicos normais (aproximadamente $1 \mathrm{~m} / \mathrm{s}$ ) e com gradiente pressórico variando de 2,5 a $4,0 \mathrm{mmHg}$ (média de $3,1 \pm 0,8 \mathrm{mmHg}$ ).

\section{COMENTÁRIOS}

A técnica extra-anatômica com interposição do tubo de Dacron entre a aorta ascendente e aorta torácica descendente via esternotomia, para correção de coarctação do arco aórtico em adultos, mostrou-se segura, de fácil execução e eficiente no alívio dos sintomas, sendo também útil em pacientes com lesões cardíacas associadas à coarctação da aorta ou com recoarctação.

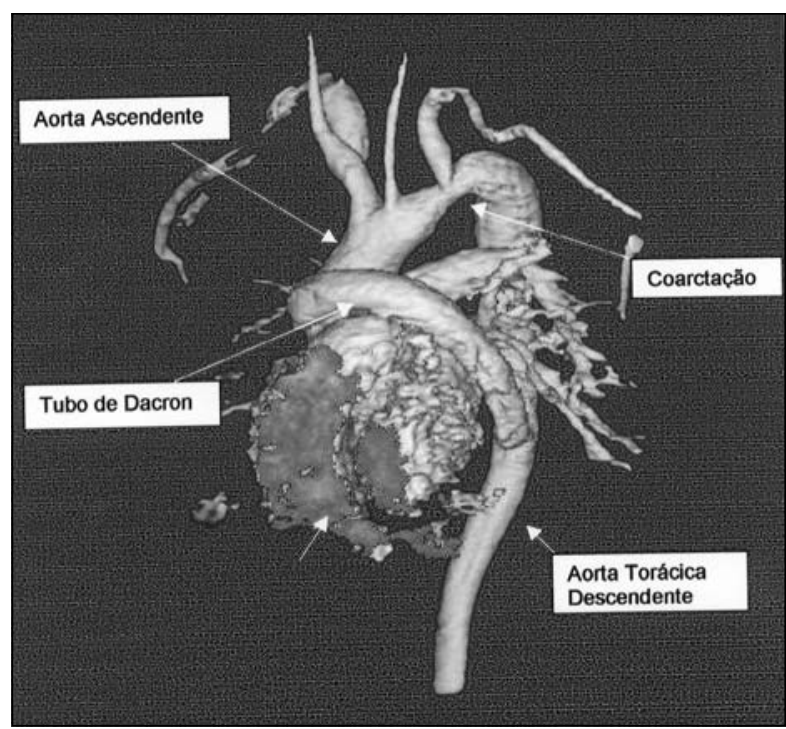

Fig. 7 - Angioressonância (pós-operatório 12 anos) de correção de coarctação distal do arco aórtico pela técnica extra-anatômica com tubo de Dacron da aorta ascendente para aorta torácica descenente, passando sobre a artéria pulmonar e lateralmente ao ventrículo esquerdo.

Preocupações com complicações neurológicas por manipulação do arco aórtico ou por isquemia medular ou complicações renais por hipoperfusão tecidual foram minimizadas com essa técnica. $O$ acompanhamento pós-operatório desses pacientes mostrou excelente evolução clínica e angiográfica a longo prazo, com ausência de reestenose e de reoperação com até 21 anos de seguimento.

A história natural da coarctação da aorta foi bem documentada no clássico trabalho de CAMPBELL ${ }^{(6)}$, onde $25 \%$ dos pacientes não operados faleceram antes dos 20 anos, $50 \%$ morreram antes dos 32 anos e $90 \%$ antes dos 58 anos de idade. Comparando-se a expectativa de vida dessa população com a população geral de mesma faixa etária, observa-se um aumento da mortalidade na $3^{\mathrm{a}}$ e $5^{\mathrm{a}}$ décadas de vida. Estudos com seguimento pós-operatório tardio mostraram os benefícios do tratamento cirúrgico da coarctação da aorta com aumento na expectativa de vida ${ }^{(7,8)}$.

Em adultos, o tratamento cirúrgico da coarctação da aorta apresenta algumas dificuldades técnicas decorrentes, principalmente, da espessura da parede da aorta, dificuldade de mobilização do arco aórtico, calcificação da aorta e da circulação colateral muito desenvolvida. O tipo de correção cirúrgica depende não só da localização e extensão da coarctação, mas também das alterações da parede da aorta.

A coarctação da aorta é mais comumente tratada com correção anatômica, tais como ressecção 
Lisboa L A F, Abreu Filho C A C, Dallan L A O, Rochitte C E, Souza J M, Oliveira S A - Tratamento cirúrgico da coarctação do arco aórtico em adulto: avaliação clínica e angiográfica tardia da técnica extra-anatômica. Rev Bras Cir Cardiovasc 2001; 16(3): 187-94.

do segmento estenótico e anastomose término-terminal ou aortoplastia com "flap" da artéria subclávia esquerda (crianças e adultos jovens) ou com ressecção do segmento estenótico e interposição de um tubo de Dacron entre a porção proximal e distal da aorta (segmentos longos e adultos). A aortoplastia, com colocação de "patch" e ampliação do segmento estenótico, também pode ser realizada, porém tem maior incidência de desenvolvimento tardio de pseudo-aneurisma $(9,10)$.

Entretanto, em algumas situações com coarctação do arco aórtico, formas atípicas de coarctação, calcificação na área coarctada ou na recoarctação o tratamento cirúrgico com correção anatômica apresenta alto risco com aumento da morbidade e mortalidade(11). Hemorragia intra-operatória, lesão do nervo laríngeo recorrente, paralisia frênica, quilotórax e infecção pulmonar são mais freqüentes quando a correção anatômica é realiza$\mathrm{da}^{(12-14)}$. A isquemia medular com seqüelas neurológicas pode ocorrer em $0,5 \%$ dos pacientes opera$\operatorname{dos}^{(15)}$, podendo chegar à 1,5\%(16). Para evitar ou diminuir as complicações decorrentes do tratamento cirúrgico das formas complexas de coarctação da aorta, onde em algumas vezes faz-se necessário a realização de hipotermia profunda e parada cardiocirculatória total, várias técnicas extraanatômicas foram propostas ${ }^{3,4}$. Em 1973, WELDON et al. ${ }^{17}$ descreveram uma técnica extra-anatômica com anastomose de um tubo entre a artéria subclávia esquerda e a aorta descendente, via toracotomia esquerda. Em 1974, SIDERYS et al. ${ }^{3}$ realizaram a interposição de um tubo entre a aorta ascendente e a aorta abdominal infra renal, realizando a esternotomia e a laparotomia, simultaneamente. Uma variação dessa técnica com anastomose distal do tubo realizada na aorta abdominal supra-celíaca, foi descrita por WUKASCH et al. ${ }^{(4)}$. A interposição do enxerto entre a aorta ascendente e abdominal infrarenal ou supracelíaca tem sido utilizada para formas atípicas de coarctação da aorta $(18,19)$. No entanto, essa técnica tem a desvantagem da necessidade do uso de um enxerto longo e da realização da laparotomia. Em 1975, EDIE et al. (20) propuseram a realização simultânea da toracotomia esquerda e da esternotomia com anastomose do tubo entre a aorta ascendente e a aorta torácica descendente, diminuindo o comprimento do enxerto, porém realizando duas incisões.

Em 1981, OLIVEIRA et al. ${ }^{(5)}$ realizaram com sucesso uma variante da técnica extra-anatômica para correção cirúrgica da coarctação do arco aórtico em adulto, interpondo um tubo de Dacron entre a aorta ascendente e aorta torácica descendente, via esternotomia e com abordagem do pericárdio posterior, simplificando a técnica. Nesse mesmo ano,
VIJAYANAGAR et al. ${ }^{(21)}$ relataram um caso onde técnica semelhante foi empregada para a correção simultânea da coarctação da aorta e da insuficiência da valva aórtica. A vantagem da técnica extraanatômica, por via transesternal é evitar a manipulação da região de coarctação aórtica onde a parede da aorta apresenta alterações estruturais e a circulação colateral é muito desenvolvida, principalmente em adultos. Pela mesma via, com abordagem do pericárdio posterior e sem a necessidade da realização de laparotomia, permite o acesso à aorta descendente logo acima do diafragma, em um segmento da aorta onde a circulação colateral é menor. Em pacientes com doenças cardíacas associadas, essas poderão ser corrigidas simultaneamente.

A utilização da circulação extracorpórea, sem a parada cardíaca e mantendo normotermia, visa facilitar a exposição da aorta descendente, elevando-se a ponta do coração, sem alteração hemodinâmica significativa e mantendo uma boa perfusão tecidual. Recentemente, KANTER et al. (22) realizaram com sucesso essa mesma técnica em 19 crianças, sendo que em $13(68,0 \%)$ casos a operação foi realizada sem circulação extracorpórea.

Complicações neurológicas, respiratórias, coagulopatias e mortalidade não têm sido observadas no pós-operatório com a utilização da técnica extra-anatômica $(23,24)$. Acompanhamentos a longo prazo têm mostrado boa evolução clínica com regressão da hipertensão arterial sistêmica e ausência de reoperações ${ }^{(25,26)}$. No entanto, a avaliação a longo prazo do enxerto de Dacron, comumente é realizada com estudo ecocardiográfico; controles angiográficos, para melhor caracterização do enxerto têm sido raramente explorados(26). Em nosso estudo, $11(73,3 \%)$ pacientes realizaram controle pós-operatório com ressonância magnética, mostrando bom funcionamento do tubo de Dacron com seguimento pós-operatório variando de 5 dias a 21 anos (média de 6,1 anos). A angiografia com ressonância magnética tem se mostrado extremamente útil, não só no seguimento pós-operatório, mas também na avaliação pré-operatória para melhor planejamento cirúrgico nas formas complexas de coarctação da aorta.

Com base na nossa experiência, concluímos que a técnica extra-anatômica com interposição do tubo de Dacron entre a aorta ascendente e a aorta torácica descendente, por via transesternal, é um procedimento relativamente simples e com baixa morbi-mortalidade. Os bons resultados a longo prazo mostram que esta técnica pode ser uma alternativa segura e menos invasiva, para a correção de coarctação do arco aórtico ou recoarctação em adultos. 
Lisboa L A F, Abreu Filho C A C, Dallan L A O, Rochitte C E, Souza J M, Oliveira S A - Tratamento cirúrgico da coarctação do arco aórtico em adulto: avaliação clínica e angiográfica tardia da técnica extra-anatômica. Rev Bras Cir Cardiovasc 2001; 16(3): 187-94.

RBCCV 44205-546

Lisboa L A F, Abreu Filho C A C, Dallan L A O, Rochitte C E, Souza J M, Oliveira S A - Surgical repair of the aortic arch coarctation in adults: long-term clinical and angiographic evaluation of the extra-anatomic aortic bypass technique. Rev Bras Cir Cardiovasc 2001; 16(3): 187-94.

ABSTRACT: Objective: We analyzed late results of extra-anatomic aortic bypass technique with Dacron graft from the ascending aorta to the descending aorta for repair of aortic arch coarctation in adults.

Material and Methods: From 1979 to 2000, a total of 15 adult patients, aged 18 years to 61 years (mean $30.8 \pm 12.1$ years) underwent extra-anatomic bypass graft for surgical repair of aortic arch coarctation. Operative exposure was median sternotomy with posterior pericardial approach in $13(86.7 \%)$ patients and left thoracotomy in $2(13.3 \%)$. Associated procedures were performed in $3(20.0 \%)$ patient and there were $4(26.7 \%)$ reoperations. The patients had clinical evaluation, echocardiographic and angiographic studies, the latter with magnetic resonance post-operative. Follow-up was $6.9 \pm 6.7$ years (range 30 days to 21 years).

Results: There was neither early or late mortality nor neurologic complications. There was no late complications with the Dacron graft neither reoperations. All patients were asymptomatic with patent Dacron graft confirmed by echocardiography. Five (33.3\%) patients had mild hypertension. The magnetic resonance was done in $11(73.3 \%)$ patients and the Dacron graft were long-term patent in all.

Conclusions: The extra-anatomic bypass aortic technique with Dacron graft from the ascending aorta to the descending aorta for repair of aortic arch coarctation in adults is a safe operation with low morbidity and mortality. The good long-term results proved to be safe and less invasive technique to repair the aortic arch coarctation or recoarctation in adults.

DESCRIPTORS: Aortic coarctation, surgery. Aorta, thoracic, abnormalities. Aortic coarctation, radiography. Aorta, thoracic, surgery. Aorta, thoracic, radiography. Coronary angiography.

\section{REFERÊNCIAS BIBLIOGRÁFICAS}

1 Backer C L \& Mavroudis C - Congenital Heart Surgery Nomenclature and Database Project: patent ductus arteriosus, coarctation of the aorta, interrupted aortic arch. Ann Thorac Surg 2000; 69(4 suppl): S298-307.

2 Crafoord C \& Nylin G - Congenital coarctation of the aorta and its surgical treatment. $J$ Thorac Surg 1945; 14: 347-61.

3 Siderys H, Graffis R, Halbrook H, Kasbeckar V - A technique for management of inaccessible coarctation of the aorta. J Thorac Cardiovasc Surg 1974; 67: 568-70.

4 Wukasch D C, Cooley D A, Sandiford F M, Nappi G, Reul Jr. G J - Ascending aorta-abdominal aorta bypass: indications, technique, and report of 12 patients. Ann Thorac Surg 1977; 23: 442-8.

5 Oliveira S A, Oliveira H A, Kedor H H, Auler Jr. J O, Souza J M - Variante técnica para reoperação de coarctação do arco aórtico. Arq Bras Cardiol 1981; 37: 395-7.

6 Campbell M - Natural history of coarctation of the aorta. Br Heart J 1970; 32: 633-40.

7 Presbitero $\mathrm{P}$, Demarie D, Villani $\mathrm{M}$ et. al. - Long-term results (15 - 30 years) of surgical repair of aortic coarctation. Br Heart J 1987; 57: 462-7.
8 Bouchart F, Dubar A, Tabley A et al. - Coarctation of the aorta in adults: surgical results and long-term followup. Ann Thorac Surg 2000; 70: 1483-9.

9 Bergdahl L \& Ljungqvist A - Long-term results after repair of coarctation of the aorta by patch grafting. $J$ Thorac Cardiovasc Surg 1980; 80: 177-81.

10 Hehrlein F W, Mulch J, Rautenburg H W, Schlepper M, Scheld $\mathrm{H} \mathrm{H}$ - Incidence and pathogenesis of late aneurysms after patch graft aortoplasty for coarctation. J Thorac Cardiovasc Surg 1986; 92: 226-30.

11 Liberthson R R, Pennington D G, Jacobs M L, Daggett W M - Coarctation of the aorta: review of 234 patients and clarification of management problems. $A m \mathrm{~J}$ Cardiol 1979; 43: 835-40.

12 Foster E D - Reoperation for aortic coarctation. Ann Thorac Surg 1984; 38: 81-9.

13 Sweeney M S, Walker W E, Duncan J M, Hallman G L, Livesay J J, Cooley D A - Reoperation for aortic coarctation: techniques, results, and indications for various approaches. Ann Thorac Surg 1985; 40: 46-9.

14 Pennington D G, Liberthson R R, Jacobs M, Scully $H$, Goldblatt A, Daggett W M - Critical review of experience with surgical repair of coarctation of the aorta. J Thorac Cardiovasc Surg 1979; 77: 217-29. 
Lisboa L A F, Abreu Filho C A C, Dallan L A O, Rochitte C E, Souza J M, Oliveira S A - Tratamento cirúrgico da coarctação do arco aórtico em adulto: avaliação clínica e angiográfica tardia da técnica extra-anatômica. Rev Bras Cir Cardiovasc 2001; 16(3): 187-94.

15 Brewer L A, Fosburg R G, Mulder G A, Verska J J Spinal cord complications following surgery for coarctation of the aorta: a study of 66 cases. $J$ Thorac Cardiovasc Surg 1972; 64: 368-81.

16 Lerberg D B, Hardesty R L, Siewers R D, Zuberbuhler $\mathrm{J}$ R, Bahnson H T - Coarctation of the aorta in infants and children: 25 years of experience. Ann Thorac Surg 1982; 33: 159-70.

17 Weldon C S, Hartmann Jr. A F, Steinhoff N G, Morrissey $J \mathrm{D}$ - A simple, safe, and rapid technique for the management of recurrent coarctation of the aorta. Ann Thorac Surg 1973; 15: 510-9.

18 Robicsek F, Hess P J, Vajtai P - Ascending-distal abdominal aorta bypass for treatment of hypoplastic aortic arch and atypical coarctation in the adult. Ann Thorac Surg 1984; 37: 261-3.

19 Di Eusanio G, Astolfi D, Piccoli G P, Clementi G, Palminiello A - Diffuse hypoplasia of the thoracic aorta: surgical treatment with ascending aortasupracaeliac abdominal aorta bypass graft. $J$ Cardiovasc Surg 1982; 23: 344-7.

20 Edie R N, Janani J, Attai L A, Malm J R, Robinson G - Bypass grafts for recurrent or complex coarctations of the aorta. Ann Thorac Surg 1975; 20: 558-66.

21 Vijayanagar R, Natarajan P, Eckstein P F, Bognolo D A,
Toole J C - Aortic valvular insufficiency and postductal aortic coarctation in the adult: combined surgical management through median sternotomy: a new surgical approach. J Thorac Cardiovasc Surg 1980; 79: 266-8.

22 Kanter K R, Erez E, Williams W H, Tam V K - Extraanatomic aortic bypass via sternotomy for complex aortic arch stenosis in children. $J$ Thorac Cardiovasc Surg 2000; 120: 885-90.

23 Grinda J M, Macé L, Dervanian P, Folliguet T A, Neveux $J$ Y - Bypass graft for complex forms of isthmic aortic coarctation in adults. Ann Thorac Surg 1995; 60: 1299-302.

24 Izhar U, Schaff H V, Mullany C J, Daly R C, Orszulak T A - Posterior pericardial approach for ascending aorta-to-descending aorta bypass through a median sternotomy. Ann Thorac Surg 2000; 70: 31-7.

25 Jacob T, Cobanoglu A, Starr A - Late results of ascending aorta-descending aorta bypass grafts for recurrent coarctation of aorta. J Thorac Cardiovasc Surg 1988; 95: 782-7.

26 Heinemann M K, Ziemer G, Wahlers T, Kohler A, Borst $H$ G - Extraanatomic thoracic aortic bypass grafts: indications, techniques, and results. Eur J Cardiothorac Surg 1997; 11:169-75. 